\title{
Environmental and Advocacy Implications of a Proposed Global "Census of the Fishes"
}

Michelle A. Duval and Douglas N. Rader Environmental Defense Fund - Raleigh, North Carolina USA

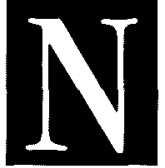

ever has there been a more apparent need for information about our ocean resources. However, devising a means of collecting and interpreting data on such a dynamic environment is a daunting task. Recognizing the need for information, the Sloan Foundation has proposed the undertaking of a global "Census of Marine Life" - i.e., a global marine biodiversity Census, and has expended much effort to examine the different aspects of such a survey. The Environmental Defense Fund (EDF) agreed to help the Sloan Foundation examine the environmental, conservation and advocacy dimensions of a Census of this nature. This paper represents a synopsis of our investigations.

To collect this information, we first established a panel of carefully selected scientists from both the academic and advocacy/environmental communities. After several phone and email conversations, we prepared a series of survey questions for the panelists, which were as follows:

1. Given technical limitations, please describe what you view as the major drawback(s) of conducting such a study.

2. Describe the major benefits or products that this study would have for the environmental/conservation/advocacy community.

3. A project of this magnitude could take on many different focuses. What do you feel should be the primary focus in terms of expanding our global knowledge of the ocean? (more precise estimates of distribution and abundance, increased understanding of certain ecological processes, focus on particular taxa, productivity, etc.)

4. What do you feel would be the best way to coordinate an effort such as this with projects that are ongoing? Would you envision an effort like this to be a primary or secondary data-collection effort?
5. Given the present levels of uncertainty in biodiversity data, do you feel that this kind of effort could produce useful, worthwhile data?

We compiled the results from the survey (available at http:/ / core.cast.msstate.edu/censhome.html) into a short draft which identified major suggestions and concerns, and posed issues for discussion. This was circulated to a number of colleagues in the environmental and advocacy communities, who were invited to participate in a 2-3 hour discussion regarding the proposed Census.

Major potential issues identified during this process included the following:

1. Minimization of direct impacts of testing and sampling on sensitive ecosystems and on individual organisms wherever possible;

2. Careful planning of data acquisition and management so as not simply to help resource users better find exploitable resources;

3. Development of broad-based support so that net resources for oceanic exploration and understanding, and for management improvements, are enhanced rather than monopolized.

Most respondents easily understood the benefits of such an endeavor. Benefits were identified in several areas:

1. Stronger science on which to base oceanic, estuarine and coastal management decisions, across-theboard;

2. Better understanding of oceanic ecosystem processes;

3. Better underpinnings for management of fisheries and other marine resource exploitation; 
4. Better identification of special places in the sea for appropriate protection and management;

5. Establishment of a baseline against which changes could be measured.

Two discussions were held July 30, 1998: one in EDF's Washington, DC office and the other in EDF's Oakland, $\mathrm{CA}$ office. The discussions were fruitful and generated many creative ideas concerning the Census. The comments from these discussions were incorporated into a draft paper, which was circulated again among panelists, discussion participants and other members of the environmental and academic communities who were unable to attend the workshops. The results of this round of comments were incorporated for the final version of the paper.

First, we present the major ideas of the environmental community on two topics: (1) What do we want/need to learn? (2) How should we go about obtaining this information?

Second, we present a summary of public responses obtained from EDF's website. We felt that it would be useful to obtain public opinion on a proposed undertaking of this magnitude, as it is quite likely that public support would be necessary for such a venture.

Finally, given the volume of data the Sloan Foundation has collected on various subtopics related to the proposed Census, we respectfully suggest a plan for moving forward with the Census, should the Sloan Foundation decide to pursue this topic.

\section{RESPONSES FROM THE ENVIRON- MENTAL/ADVOCACY COMMUNITY}

\section{What do we want to learn?}

It was strongly agreed upon that an ecosystem approach to the Census is necessary. The greatest potential of this project is that the sum of its parts could be greater than the whole. Currently, there is little support in scientific journals for the broad scale synthesis that a Census implies. However, the formation of useful hypotheses is critical to the success of the Census. The project needs to maintain a focus on understanding ecosystem processes (such as recruitment, fecundity, life cycles, etc.) in the marine realm and how different ecosystems within that environment relate to one another. There is a great need among members of the conservation community for this information in order to strategically plan protection efforts. Within that context, a number of suggestions were made:

- Indicators of ecosystem health: A focus on the processes which structure and control ecosystems and the relationships between them would allow us to identify "ecosystem indicators"-proxies that can be used to monitor the health of the ocean and establish thresholds for concern. The Sloan Foundation has stated that a goal of this project would be to leave in place some type of infrastructure or system for continued global monitoring efforts. Characterization of the indicators of ecosystem health would not only fulfill this purpose, but also provide accountability for ocean-related activities. Ecosystem indicators can help shift the burden of proof with respect to human impact on the ocean realm.

- Representative ecosystems: The resources required to carry out a comprehensive ocean survey are immense, and the cooperation required from other countries would be significant. It was suggested that focusing on a few representative marine ecosystems - coral/live hard-bottoms, benthic continental shelf, pelagic continental shelf, abyssal plain, deep-sea vents, intertidal, estuarine - rather than the entire ocean, would allow a more thorough understanding of the processes affecting the distribution and abundance of species within those ecosystems. This approach also provides for identification of indicators of ecosystem health, as well as a determination of the impact of human activities on ocean ecosystems. Finally, studying an ecosystem is likely to produce data that will be more accurate (and for a longer period of time) than abundance data, given the vagaries of population dynamics. Species abundance numbers can fluctuate from year to year and in response to poorly documented processes and variables. Concentrating valuable intellectual, temporal and financial resources in this manner will provide a set of comprehensive information.

- Marine reserves: The Census provides an excellent opportunity to use marine reserves as a tool. Reserves can be used to document the human impacts on various ecosystems, and can serve as baseline areas, or scientific "controls" amidst the "experiment" of activities that take place today. This includes both fishing and non-fishing impacts, as well as ocean and land-based activities. Setting up a network of reserves that captures specific, unimpacted ecosystems, examining the processes that drive these ecosystems, and comparing them to the processes operating in areas highly affected by human activity can provide evidence for or against single-species management. In the same regard, both light and heavily fished areas could be sampled in order to provide a gradient of comparison for disturbed and undisturbed systems.

- Human impacts: A "theme" in the above suggestions is determining the impact of human activities on ocean ecosystems. Whatever the focus and hypotheses of the Census turn out to be, human effects should be incorporated, as many people will 
wish to use the data coming out of the project to make management decisions in this regard.

As a final comment, it should be realized that a project of this magnitude, which seeks to provide an incredibly large and valuable amount of scientific information, will automatically have many non-science implications. These implications should be considered when designing the project, so that unfair exploitation of data does not occur. Managers, advocates, special interest groups, etc. will be eager to use the results of the Census, therefore it is of the utmost importance to ensure that the information collected is unbiased and made readily available to all.

\section{How do we go about learning this?}

- Establish a governance structure: structure would need to be created in order to both direct the study and handle the volumes of data gathered from the project. It was suggested that this be in the form of one or more international panels, perhaps modeled after one of the already established international studies, such as ICES (International Council on Exploration of the Sea). The panel(s) should be diverse and bring in stakeholders from many different arenas: conservation, academia, fishing industry, tourism, etc. However, it should be maintained above all else that the goal of the project is to bring in accurate science.

- Project design: Establish a panel whose task is to determine the priorities, focus, and hypotheses of the project, and then issue a general request for proposals. Taking this approach allows people/groups most interested in participating to come forth with their best and most creative ideas, some of which may not have been previously considered by either the Sloan Foundation or the panel. A second panel should be established to review proposals for their scientific integrity, conformity with the hypotheses being tested, and how well component proposals fit together to accomplish the overall goal of the project.

- Information management: Data acquisition needs to be coupled with a structure for using it. A panel needs to be appointed to develop a method to handle the collection and use of data generated from the project. Data should be collected in a uniform manner so that they may be analyzed easily. A central repository, perhaps on the Internet, should be created. Allowing access to both raw and processed (published) data via a website would generate creative analyses and aid in putting off disputes over data rights.
- Existing infrastructure: A thorough examination of existing research efforts and global databases (such as the United Nations Food and Agriculture Organization [FAO] fisheries statistics and cooperative statistics programs among the U.S. federal fishery management councils) needs to be undertaken. This includes investigating the possibility of employing military vessels for cruises.

- Public involvement: It is important to engage public support in an effort of this magnitude, especially if public funds are used in some measure to fund the project. The greater the support, the less the feeling of cost to the general public. Focus groups might be formed to explain the focus and hypotheses of the project. Public aquaria could provide a public information source on the status of the project and perhaps even a "live link" to field operations. It might also be beneficial to change the title of the project from "Census of Marine Life" to something more indicative of what it encompasses, such as "Global Ocean Discovery Program." A more appropriate title would serve to dispel somewhat the objections of animal rights activists, and perhaps engage greater public support.

\section{RESPONSES FROM THE PUBLIC}

As mentioned previously, we solicited responses from the general public via EDF's website. To this effect, we placed background material, the survey questions listed above and an explanation of EDF's role in the process on our website and asked users to express their thoughts. We received approximately 30 responses, most of which were enthusiastically supportive of the project and ranged from simply "This is a great idea, please do it" to very detailed answers to the survey questions. The respondents came from a broad range of occupations: doctors, lawyers, scientists, fishermen, students, parents, etc. Concerns and sentiments expressed included:

- Funding: Where would the money come from to pay for the project? Would it be taken from ongoing research efforts?

- Cooperation: There was concern that complete and unbiased cooperation might be difficult to obtain from major commercial fishing nations that would only be interested in information for exploitative purposes. The idea of an international committee composed of member nations that would be co-managed by a business and scientific manager was suggested, so that individual countries could participate in funding measures and building public support. 
- Focus/questions to be addressed: There were many comments on this topic, with respondents expressing an interest that the Census cover not only inventory of species, but also ecosystem processes and how they are related to various human/environmental impacts on the ocean (e.g., pollution, habitat change, global warming). It was also mentioned that there needs to be a "context" within which the results of the Census could be interpreted.

- Fisheries management: There were many remarks from citizens, commercial and recreational fishermen expressing frustration with current management and knowledge of fish stocks, and hopes that a Census could eliminate uncertainties and allow more responsible fishing. A few fishermen offered to help with data collection by installing dataloggers on vessels, etc.

\section{PLAN OF ACTION}

The following is a suggestion for how the Sloan Foundation might proceed in its pursuit of a global "fish census." These recommendations attempt to take into account the logistical difficulties that may be encountered.

1. Complete a thorough review and analysis of existing information and information-gathering processes focusing on the world ocean, including literature, databases, academic institutions, federal agencies, the military, etc. to determine the scope of information that is already available or being collected and, most importantly, where critical gaps in knowledge lie. This exercise will be essential to laying out the overall goals and hypotheses of the project. This process of review could possibly take six months to one year to complete.

2. Form a high-visibility, international management board, with equitable representation of ocean "interests," as well as a pair of international advisory panels, one of scientists (academic and agency), and the other of ocean "users": fishermen, environmentalists, citizens, and other user groups such as freight operators, charter boat captains, business leaders, statisticians, etc. Care should be taken to include representation from all user groups and interested nations on the "interest" advisory panel, and an equitable balance of interests on the management board. It should be emphasized that the goal of the project is scientific, not political. Panel formation may take one to three months, depending on the ability to schedule and contact people.
3. Once the panels are established, face-to-face meetings should be convened. A series of pre-meeting communications via e-mail or conference call should be arranged to stimulate thoughts on composition of panels and division of labor. This process could take up to six months.

4. The management board, with advice from the advisory panels, should determine the goals, priorities and hypotheses of the project, and then solicit a set of proposals from the global scientific community to address the specific questions at hand. This panel should also be responsible for developing criteria for review of proposals. The scientific advisory panel could then review proposals for scientific integrity and value in light of the specified goals of the project; the "interest" advisory panel would provide advice to the management board on project selection. This part of the process could take up to two years.

5. Establish a robust system for information management, designed by experts, with special attention to methods of data acquisition, a format for storing data, means of analysis and a process for users to access data. This could be quite time-consuming, and beyond the experience of the international panels, hence the suggestion to contract this task out to professionals. Information developed under the program should be as widely available as possible.

6. Once data collection processes are underway, convene periodic meetings, whether in person or via telephone, of the management board and advisory committees to evaluate progress on components of the overall project and determine if any alterations in data collection or sampling methods are necessary. Five years will probably be needed for data collection to be completed for components of the project.

7. Information dissemination. Obviously, numerous individual, peer-reviewed publications will come out of this project. However, production of a white paper for public review and a website devoted to the project would be beneficial in incurring public support for this and future endeavors. Creation of a website need not necessarily be an end product, but could be ongoing, with periodic updates as data and analyses are completed. 